\title{
Signs and Symptoms of Acoustic Neuroma at Initial Presentation: An Exploratory Analysis
}

Robert W. Foley ${ }^{1}$, Shahram Shirazi ${ }^{2}$, Robert M. Maweni ${ }^{2}$, Kay Walsh ${ }^{3}$, Rory McConn Walsh ${ }^{4}$, Mohsen Javadpour ${ }^{5}$, Daniel Rawluk ${ }^{5}$

1. Department of Neurosurgery, Addenbrooke's Hospital, Cambridge University Hospitals, United Kingdom 2. School of Medicine, University College Dublin 3. Department of Skull Base Surgery, Beaumont Hospital, Dublin, Ireland 4. Department of Otolaryngology, Beaumont Hospital, Dublin, Ireland 5. Department of Neurosurgery, Beaumont Hospital, Dublin, Ireland

Corresponding author: Robert W. Foley, rwjfoley@gmail.com

\section{Abstract}

\section{Introduction}

The objective of this study was to describe the most common clinical features associated with an acoustic neuroma diagnosis and to identify those features associated with larger tumour size at initial diagnosis.

\section{Methods}

The clinical information of 945 consecutive patients diagnosed with acoustic neuroma at a single centre between 1992 and 2015 was analysed. Clinical features were examined and the relationship between these features and tumour size $(>2.5 \mathrm{~cm})$ was analysed using descriptive statistics and logistic regression analysis. Statistical analysis was performed in R version 3.1.1.

\section{Results}

The most common presenting symptom was a unilateral hearing loss in 752 patients (80\%), with a progressive pattern in $90 \%$ of these cases. The second most common presenting symptom was unilateral tinnitus, accounting for $6.3 \%$, while ataxia, vertigo and headache accounted for $3.8 \%, 3.4 \%$ and $2 \%$, respectively. The diagnosis of acoustic neuroma was an incidental finding in 20 patients (2.1\%). Temporal analysis demonstrated a downward trend in the number of patients presenting with hearing loss and an increased proportion of patients presenting with other symptoms. On multivariate analysis, larger tumour size was associated with abnormal tandem gait (odds ratio 8.9, $\mathrm{p}=0.02$ ), subjective facial weakness (odds ratio $5.3, \mathrm{p}<0.001$ ), abnormal facial sensation on examination (odds ratio $3.0, \mathrm{p}=0.03$ ) and headache (odds ratio $2.6, \mathrm{p}<0.001)$.

\section{Conclusion}

Received 08/14/2017 Review began 09/10/2017 Review ended 11/13/2017 Published 11/15/2017

\section{(c) Copyright 2017}

Foley et al. This is an open access article distributed under the terms of the Creative Commons Attribution License CC-BY 3.0., which permits unrestricted use, distribution, and reproduction in any medium, provided the original author and source are credited.
The majority of patients with acoustic neuroma present with the classic, progressive, unilateral hearing loss. However, the pattern of presentation in acoustic neuroma patients is changing. Features in the history indicative of a larger tumour are headaches and subjective facial weakness, whilst concerning features on examination are abnormal tandem gait and altered facial sensation.

\section{Categories: Otolaryngology, Neurosurgery, Epidemiology/Public Health}

Keywords: acoustic neuroma, diagnosis, epidemiology, signs and symptoms

\section{Introduction}

Acoustic neuroma, or vestibular schwannoma, is a benign tumour of the Schwann cell that most commonly arises from the vestibular nerve [1]. Acoustic neuroma is one of the most common intracranial tumours and has been reported to make up approximately $6 \%-10 \%$ of tumours in most series [2]. Acoustic neuroma has an incidence of approximately 12 per million in the United States of America and approximately 20 per million in a contemporary European cohort [3-4]. However, the incidence of acoustic neuroma is increasing [5-6]. An analysis of 26 years worth of prospectively collected data, from 1976 to 2002 in Denmark, has shown the incidence move from 7.8 per million to 19.3 million [5]. In tandem with these changes, there are increasing numbers of smaller tumours being diagnosed. The Danish data has shown a size decrease from an average of $30 \mathrm{~mm}$ to an average of $10 \mathrm{~mm}$ in the period from 1976 to 2008. These trends in the epidemiology of acoustic neuroma may mean that the presentation of patients suffering from this disease may also be changing. Thus, it would be of benefit for healthcare providers, be they in primary care, a hospital setting or a surgical sub-specialty, to be aware of any emerging patterns in the presentation of acoustic neuroma patients. Descriptions of the signs and symptoms of acoustic neuroma within the literature are largely from older studies, which may not be applicable to contemporary practice [7-8]. Furthermore, adequate statistical analysis of these variables with multivariable regression has yet to be performed. 
Therefore, the objectives of this study were to describe the most common clinical features associated with the initial presentation of patients with acoustic neuroma and to examine the temporal trends in these features. Within the acoustic neuroma management paradigm, larger tumours are an important clinical entity, as they require earlier definitive treatment. The study of clinical and historical features within each patient's presentation and the identification of the features associated with larger tumours will allow for an accurate diagnosis to be made quickly and, therefore, the appropriate investigations can be targeted towards those patients with the greatest clinical need. Hence, a secondary objective of this study was to identify those clinical features associated with larger tumour size at initial diagnosis.

\section{Materials And Methods}

All patients within the cohort were diagnosed with acoustic neuroma and their data collected prospectively and added to a database. The present study analyses the data of all patients within the database from its inception in 1992 until 2015. Each patient was referred to the national neurosurgical centre at Beaumont Hospital, Ireland, and underwent a thorough history and examination, which was recorded in the database. Clinical features in the history and examination are reported by the number of patients and the percentage, with all percentages rounded to the nearest whole number. Tinnitus severity was recorded as mild, moderate or severe, as per Baguley et al. [9]. Facial nerve function was determined using the House-Brackmann grading scale [10]. Tumour size was measured based on each patient's initial magnetic resonance imaging (MRI) scan and is the extrameatal maximum diameter of the tumour. Size was recorded in one of five categories, namely, $<1.5 \mathrm{~cm}, 1.5-2.4 \mathrm{~cm}, 2.5-3.4 \mathrm{~cm}, 3.5-4.4 \mathrm{~cm}$ and $>4.5 \mathrm{~cm}$.

In order to analyse those patients with larger tumours, tumour size was dichotomised as those less than 2.5 $\mathrm{cm}$ and those $2.5 \mathrm{~cm}$ or greater. The relationship between patient clinical characteristics and tumour size was analysed by univariable and multivariable logistic regression. Odds ratios (OR) and 95\% confidence intervals were calculated and a $\mathrm{p}$ value of $<0.05$ is considered statistically significant. Time series analysis took place via the statistical package "TTR" and data was smoothed using simple moving averages. A statistical analysis was carried out using R v0.98 and the relevant statistical packages.

\section{Results}

The study cohort consisted of 945 consecutive patients diagnosed with acoustic neuroma. In this cohort, 752 patients (80\%) presented with unilateral hearing loss. The majority presented with a progressive hearing loss, which accounted for $90 \%$ of these patients. A sudden unilateral hearing loss was the second-most common overall presenting complaint, with 72 patients presenting in this way. Tinnitus was the main complaint in 60 patients (6\%). Acoustic neuroma was an incidental finding in 20 patients within this cohort. The other presenting complaints and the proportion of patients are demonstrated in Table 1 . 


\section{Cureus}

\begin{tabular}{|c|c|c|}
\hline & $\mathrm{n}$ & $\%$ \\
\hline Hearing Loss & 752 & 80 \\
\hline - Progressive Hearing Loss & 680 & 72 \\
\hline - Sudden Hearing Loss & 72 & 8 \\
\hline Tinnitus & 60 & 6 \\
\hline Ataxia & 36 & 4 \\
\hline Vertigo & 32 & 3 \\
\hline Asymptomatic / Incidental & 20 & 2 \\
\hline Headache & 19 & 2 \\
\hline Facial Numbness & 17 & 2 \\
\hline Otalgia & 2 & $<1$ \\
\hline Facial Pain & 1 & $<1$ \\
\hline Seizure & 1 & $<1$ \\
\hline Syncope & 1 & $<1$ \\
\hline
\end{tabular}

\section{TABLE 1: Main presenting symptom in the total cohort $(n=945)$}

The number of patients presenting with each symptom - as the main presenting complaint - is illustrated, as is the percentage this represents within the total cohort.

In terms of overall symptoms that patients were suffering from, not only the main presenting symptom, hearing loss was present in 868 patients (92\%), with 91\% complaining of a progressive course. A total of 479 patients (51\%) complained of tinnitus, 388 patients (81\%) intermittently and 91 patients (19\%) with constant symptoms. In terms of tinnitus severity, 330 patients (68\%) suffered from mild tinnitus, 144 (30\%) from moderate and $11(2 \%)$ from severe tinnitus. Headache was a historical feature in 114 patients (12\%), with $61 \%$ of these generalised and $39 \%$ focal in location. A separate pain was described in 40 patients, with a mastoid ache in 32 and otalgia in eight patients. A total of 234 patients (25\%) complained of unsteadiness when questioned. Other symptoms included facial weakness in 75 patients, facial numbness in 26 patients, blurred vision in 20 patients and dry eye in five patients. Altered sensations of the tongue and of taste were present in 15 and 13 patients, respectively. On examination, the vast majority of patients, 916 (97\%), were House-Brackmann grade I, with 20 grade II patients, two grade III, two grade IV, one grade V and four grade VI. An abnormal trigeminal sensation on examination was present in 29 patients. An examination of the fundi revealed mild disc blurring in eight patients. Gait testing revealed abnormalities of tandem gait in 54 patients (6\%).

Over the 23 years of the study period, there have been changes in the pattern of patient presentation. The proportion of patients complaining of hearing loss as the main presenting feature, overall, is decreasing (Fig. 1A). There is a clear downward trend for presentation with hearing loss in acoustic neuroma patients in the last decade. Whilst hearing loss accounted for the vast majority of presentations in the late 1990s and early 2000s, since then there has been increasing variability in the presenting symptom, with ataxia, vertigo and incidental findings accounting for more patient's presentations (Fig. 1B). The number of patients in Ireland with acoustic neuroma is increasing steadily each year, with 20 patients on average at the start of the study period, moving to greater than 60 patients each year in the later years. The average period from symptom onset to clinical presentation was 36 weeks in this study cohort. A time series analysis demonstrated no trend in time to presentation over the course of the study period. However, patients with sudden hearing loss presented in an average of 12.1 weeks, which was significantly faster $(\mathrm{p}<0.01)$ than those with a progressive hearing loss, who presented on average within 45.6 weeks. 


\section{Cureus}

A

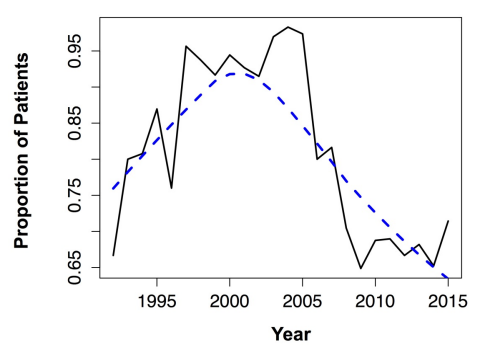

B

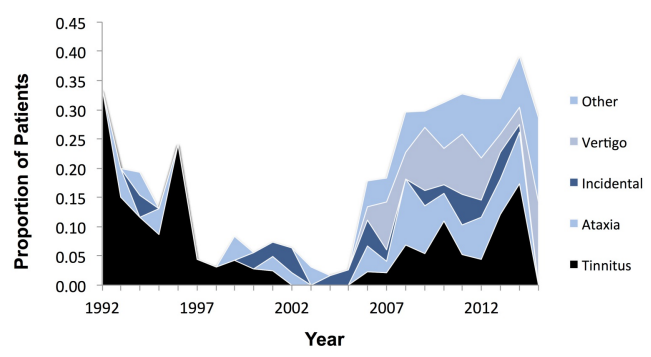

FIGURE 1: Trends in initial presentation with regards to unilateral hearing loss (A) and primary presenting symptom (B)

(A) The proportion of patients presenting with hearing loss as the main presenting symptom is shown on the $x$-axis (e.g., 0.75 corresponding to $75 \%$ of patients). The blue dashed line shows the smoothed pattern over time following a time series analysis.

(B) The trend in presenting symptoms other than hearing loss. This demonstrates that in the later years of the study (right side of the graph), there has been an increase in patients presenting with ataxia, vertigo and incidental tumours.

Tumour size within the study cohort was split into four categories, as illustrated in Fig. $2 \mathrm{~A}$. Tumours less than $1.5 \mathrm{~cm}$ in size accounted for $48 \%$ of all tumours, with $25 \%$ between $1.5-2.4 \mathrm{~cm}, 13 \% 2.5-3.4 \mathrm{~cm}, 5 \% 3.5-$ $4.4 \mathrm{~cm}$ and $4 \%$ greater than $4.5 \mathrm{~cm}$. The trend in tumour size at diagnosis is clear in this cohort. The number of smaller tumours $(<2.5 \mathrm{~cm})$ is increasing, while those $>2.5 \mathrm{~cm}$ in size are decreasing year on year (Fig. $2 B$ ). Subsequently, fewer patients were treated with a primary surgical treatment strategy, with $>80 \%$ on average treated surgically at the beginning of the study period, compared to $<20 \%$ in the last seven years of the study period.

A

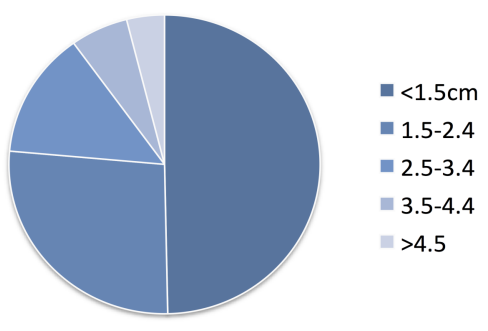

B

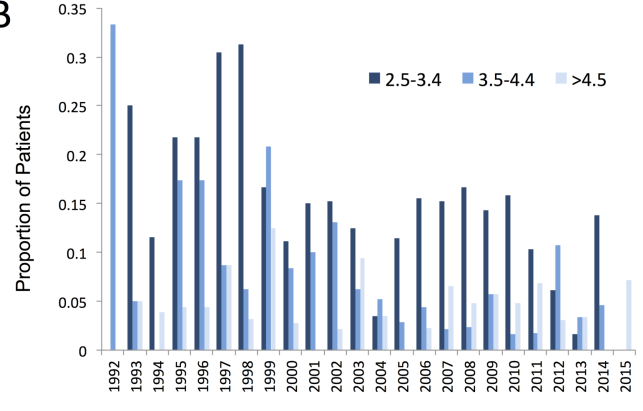

Year

FIGURE 2: Tumour size in the total cohort $(\mathrm{A})$ and the trend in the proportion of tumours within the larger size categories (B)

The breakdown of larger tumour sizes (i.e., $>2.5 \mathrm{~cm}$ ) is shown in $(\mathrm{B})$, with a decreasing trend in these larger tumours evident over the study period.

A univariable and multivariable regression analysis was undertaken to examine the clinical features most associated with a larger tumour size at diagnosis. A large number of factors was associated with larger tumour size on univariate analysis. This included taste alteration (OR 5.34, 95\% CI: 1.76-17.83; p<0.01), visual alterations (OR 9.60, 95\% CI: p<0.01), a positive Romberg's test (OR 6.05, 95\% CI: 2.07-19.9; $\mathrm{p}<0.01$ ) and dysdiadochokinesis (OR 6.31, 95\% CI: 2.55-16.99; $<<0.01$ ). However, there were only four features that were significant predictors of tumour size on both univariable and multivariable analyses (Table 2). These are both historical features and examination features. On a multivariate analysis, larger tumour size was associated with abnormal tandem gait (OR 8.9), subjective facial weakness (OR 5.3), abnormal facial sensation on examination (OR 3.0) and the presence of headache (OR 2.6). Factors that did not discriminate between smaller and larger tumour size were the presence of hearing loss (OR 0.98, $\mathrm{p}=0.95$ ), sudden versus progressive pattern of hearing loss (OR $0.93, \mathrm{p}=0.82)$, time until presentation (OR $0.99, \mathrm{p}=0.14$ ) and severity of tinnitus (OR 2.12, $\mathrm{p}=0.68$ ). 
patients experienced none of these classical symptoms. The most common symptom experienced in this atypical group was facial numbness $(24 \%)$. A case series $(n=9)$ of atypical acoustic neuroma presentations also found that facial numbness was the most common presenting symptom in $22 \%$ of patients [16]. Clinicians should, therefore, be aware of this as a potential, albeit unusual, presenting symptom of acoustic neuroma. In other words, in the absence of the classic disturbance to the vestibulocochlear nerve, the next most-common disturbance is of trigeminal nerve function. Interestingly, no patient in our cohort presented with altered facial nerve function without also suffering from vestibulocochlear nerve dysfunction.

Tinnitus is a significant symptom in acoustic neuroma patients. The relationship between tinnitus and tumour size was investigated by Baguley et al [17], who demonstrated that patients with larger tumour sizes were less likely to experience tinnitus, and this finding was statistically significant [17]. This echoes our own results on univariate analysis. However, the authors did not go on to investigate this relationship on multivariate analysis, taking into account all other symptoms. On multivariate analysis in our cohort of patients, tinnitus presence or absence was no longer a statistically significant predictor of tumour size. Although not analysed in the present study, the duration of tinnitus prior to diagnosis has also been demonstrated in a large case series to be inversely related to tumour size, with longer duration of tinnitus associated with smaller tumours [15].

The incidence of sudden hearing loss in acoustic neuroma is variable and is reported in the literature with incidences from $5 \%$ to $22 \%$ [18]. The incidence of sudden hearing loss was $12 \%$ in a case series from Moffat et al. [18], with $85 \%$ complaining of this as the main presenting symptom. Sudden hearing loss was found in 76 patients (8\%) within the present article's cohort, and 72 of these patients complained of this as their principal presenting symptom. This finding was relevant, as it was associated with a shorter time to presentation compared to those patients with progressive hearing loss. In the paper from Moffat et al., the average time to presentation was 32 months in the sudden hearing loss group compared to 40 months in those with progressive hearing loss. These findings are supported by this study, with patients suffering from sudden hearing loss presenting significantly quicker than those with a progressive pattern. The length of time to presentation is an important factor in tumour size, as longer length of symptom duration is associated with larger tumours [7]. As with Moffat et al., there was no association found between hearing loss pattern and tumour size in the present study.

Moffat et al. have reported ten percent of patients with acoustic neuroma as having an atypical main presenting symptom, i.e., not presenting with hearing loss, imbalance or tinnitus [8], much the same figure as in the present study. Patients with these atypical presentations were also reported to be more likely to have larger tumours, perhaps because of a delay in investigation [8]. This finding was not replicated in the present study, which may reflect the increasing availability of MRI, and indeed a lower threshold for MRI use. The number of patients with tumour sizes of $2.5 \mathrm{~cm}$ or greater was much larger in the Moffat et al. cohort, at $45 \%$, compared to $22 \%$ in our Irish cohort.

The number of incidental findings of acoustic neuroma appears to be low. Lin et al. [19] carried out an analysis of 505 patients and suggested that only eight of these patients underwent an incidental diagnosis. This equates to a prevalence of incidental acoustic neuromas of $1.6 \%$, which is in keeping with the prevalence in the present article. Our study revealed an incidental diagnosis in 20 patients of the 945 patients, for a prevalence of $2.1 \%$. The literature on this topic has identified a prevalence of incidental acoustic neuroma, ranging from $0 \%-2.4 \%$ [20]. Importantly, the number of patients being diagnosed with acoustic neuroma incidentally appears to be increasing. In the present study, half of the incidental cases had occurred in the last six years of the study period. This increasing number of incidental tumours represent an important clinical entity because although these tumours are generally smaller and require less intervention than symptomatic tumours [21], their growth pattern and natural history are still not elucidated. Indeed, some of the "incidental" findings of acoustic neuroma published elsewhere were found to have longstanding symptoms when questioned further [19]. In the present study, eight of the 20 incidental findings were found to have symptoms of hearing loss, tinnitus or imbalance on further questioning. Thus, the approximation for the incidence of truly atypical presentations in this cohort is $1.2 \%$ and, therefore, the number of patients with no experience of the typical symptoms of acoustic neuroma is very small. This emphasises the important role of clinical history, particularly within the primary care setting.

We recognise some limitations to this study. Only the original clinical presentation of these patients has been analysed and we have not examined the trends in these symptoms over time. Also, the tumour size presented in this study is the size at original presentation and, therefore, again, changes in size over time have not been measured. Furthermore, with regard to the self-reported length of time that symptoms have been present before presentation, this variable is subject to recall bias. One interesting variable, which may be in some way responsible for the increasing number of acoustic neuroma diagnoses, is the changing demographics of the population of Ireland. Within the timespan of the present study, the population of Ireland has grown considerably and the population is less ethnically homogenous than in the past. Unfortunately, racial ethnicity was not a recorded variable and could not be analysed.

\section{Conclusions}

The majority of patients with acoustic neuroma present with the classic, progressive, unilateral hearing loss. 
Atypical presentations and incidental findings are becoming more common, highlighting the need for a thorough history and physical examination. Features in the history indicative of a larger tumour size at diagnosis are headache and subjective facial weakness, whilst concerning features on examination are abnormal tandem gait and altered facial sensation.

\section{Additional Information \\ Disclosures}

Human subjects: Consent was obtained by all participants in this study. Beaumont Hospital Ethics (Medical Research) Committee issued approval. Animal subjects: All authors have confirmed that this study did not involve animal subjects or tissue. Conflicts of interest: In compliance with the ICMJE uniform disclosure form, all authors declare the following: Payment/services info: All authors have declared that no financial support was received from any organization for the submitted work. Financial relationships: All authors have declared that they have no financial relationships at present or within the previous three years with any organizations that might have an interest in the submitted work. Other relationships: All authors have declared that there are no other relationships or activities that could appear to have influenced the submitted work.

\section{References}

1. DeLong M, Kirkpatrick J, Cummings T, Adamson D: Vestibular schwannomas: Lessons for the neurosurgeon: part II: molecular biology and histology. Contemp Neurosurg. 2011, 33:1-4. 10.1097/01.CNE.0000409881.15693.56

2. Hoffman S, Propp JM, McCarthy BJ: Temporal trends in incidence of primary brain tumors in the United States, 1985-1999. Neuro Oncol. 2006, 8:27-37. 10.1215/\$1522851705000323

3. Babu R, Sharma R, Bagley JH, Hatef J, Friedman AH, Adamson C: Vestibular schwannomas in the modern era: epidemiology, treatment trends, and disparities in management. J Neurosurg. 2013, 119:121-130. 10.3171/2013.1.JNS121370

4. Stangerup S-E, Tos M, Thomsen J, Caye-Thomasen P: True incidence of vestibular schwannoma? Neurosurgery. 2010, 67:1335-1340.10.1227/NEU.0b013e3181f22660

5. Stangerup S-E, Tos M, Caye-Thomasen P, Tos T, Klokker M, Thomsen J: Increasing annual incidence of vestibular schwannoma and age at diagnosis. J Laryngol Otol. 2004, 118:622-627. 10.1258/0022215041917989

6. Stangerup S-E, Caye-Thomasen P, Tos M, Thomsen J: The natural history of vestibular schwannoma . Otol Neurotol. 2006, 27:547-552. 10.1097/01.mao.0000217356.73463.e7

7. Wiegand DA, Fickel V: Acoustic neuroma--the patient's perspective: subjective assessment of symptoms, diagnosis, therapy, and outcome in 541 patients. Laryngoscope. 1989, 99:179-187. 10.1288/00005537198902000-00010

8. Moffat DA, Baguley DM, Beynon GJ, Da Cruz M: Clinical acumen and vestibular schwannoma. Am J Otol. 1998, 19:82-87.

9. Baguley DM, Moffat DA, Hardy DG: What is the effect of translabyrinthine acoustic schwannoma removal upon tinnitus?. J Laryngol Otol. 1992, 106:329-331. 10.1017/S0022215100119413

10. House JW, Brackmann DE: Facial nerve grading system. Otolaryngol Neck Surg. 1985, 93:146-147. $10.1177 / 019459988509300202$

11. Kleijwegt M, Ho V, Visser O, Godefroy W, van der Mey A: Real incidence of vestibular schwannoma? Estimations from a national registry. Otol Neurotol. 2016, 37:1411-1417. 10.1097/MAO.0000000000001169

12. Mackeith SAC, Kerr RS, Milford CA: Trends in acoustic neuroma management: a 20-year review of the Oxford skull base clinic. J Neurol Surg B Skull Base. 2013, 74:194-200. 10.1055/s-0033-1342919

13. Tan M, Myrie OA, Lin FR, Niparko JK, Minor LB, Tamargo RJ, Francis HW: Trends in the management of vestibular schwannomas at Johns Hopkins 1997-2007. Laryngoscope. 2010, 120:144-149. 10.1002/lary.20672

14. Chen DA: Acoustic neuroma in a private neurotology practice: trends in demographics and practice patterns. Laryngoscope. 2017, 117:2003-2012. 10.1097/MLG.0b013e3181373876

15. Matthies C, Samii M: Management of 1000 vestibular schwannomas (acoustic neuromas): clinical presentation. Neurosurgery. 1997, 40:1-9. 10.1097/0006123-199701000-00001

16. Coca Pelaz A, Rodrigo JP, Llorente JL, Gómez JR, Suárez C: Unusual clinical presentations of vestibular schwannomas [Article in Spanish]. Acta Otorrinolaringol Esp. 2008, 58:187-189.

17. Baguley DM, Humphriss RL, Axon PR, Moffat DA: The clinical characteristics of tinnitus in patients with vestibular schwannoma. Skull Base. 2006, 16:49-58. 10.1055/s-2005-926216

18. Moffat DA, Baguley DM, von Blumenthal H, Irving RM, Hardy DG: Sudden deafness in vestibular schwannoma. J Laryngol Otol. 1994, 108:116-119. 10.1017/S0022215100126052

19. Lin D, Hegarty JL, Fischbein NJ, Jackler RK: The prevalence of "incidental" acoustic neuroma . Arch Otolaryngol Head Neck Surg. 2005, 131:241-244. 10.1001/archotol.131.3.241

20. Schmidt RF, Boghani Z, Choudhry OJ, Eloy JA, Jyung RW, Liu JK: Incidental vestibular schwannomas: a review of prevalence, growth rate, and management challenges. Neurosurg Focus. 2012, 33:4. 10.3171/2012.7.FOCUS12186

21. Jeyakumar A, Seth R, Brickman TM, Dutcher P: The prevalence and clinical course of patients with “incidental” acoustic neuromas. Acta Otolaryngol. 2007, 127:1051-1057. 10.1080/00016480701200210 